\title{
A Kind of Biomolecular Probe Sensor Based on TFBG Surface Plasma Resonance
}

\author{
Mengwei XUE ${ }^{1}$, Qi JIANG ${ }^{1 *}$, Chengjia ZHANG ${ }^{2}$, and Jianqiang LIN $^{2}$ \\ ${ }^{1}$ School of Control Science and Engineering, Shandong University, Jinan, 250061, China \\ ${ }^{2}$ State Key Lab of Microbial Technology, School of Life Science, Shandong University, Jinan, 250100, China \\ *Corresponding author: Qi JIANGＥ-mail: jiangqi@sdu.edu.cn
}

\begin{abstract}
This paper reports the application of the biomolecular probe sensor based on the tilted fiber Bragg grating (TFBG) surface plasma resonance (SPR) which can recognize the specificity of the specific molecule by depositing sensitive biological membrane outside the active golden layer. The method of self-assembly was used to make the fixed sensitive biological membrane to achieve the best effect in the experiment. To illustrate the specific recognition of the DNA molecule, the TFBG-SPR biosensor was exposed to complementary DNA solutions with the concentration of $0.1 \mathrm{mmol} / \mathrm{L}$ and $0.05 \mathrm{mmol} / \mathrm{L}$, respectively. The resonance wavelength of the TFBG-SPR biosensor increased gradually, indicating that the hybridization with the complementary DNA molecules changed the effective refractive index in the vicinity of the golden layer. Furthermore, the results illustrated the feasibility of the biomolecular probe sensor based on the TFBG surface plasma resonance for detecting the specific molecule.
\end{abstract}

Keywords: TFBG, nanostructure golden layer, surface plasma resonance, biosensor

Citation: Mengwei XUE, Qi JIANG, Chengjia ZHANG, and Jianqiang LIN, “A Kind of Biomolecular Probe Sensor Based on TFBG Surface Plasma Resonance," Photonic Sensors, 2015, 5(2): 102-108.

\section{Introduction}

After decades of development, surface plasma resonance (SPR) biosensors have become an important sensing technique in the fields of bioscience and medicine screening. Safety detection of food and medicine, genetic analysis, imunoanalysis, and protein detection are among the hot spots of the current SPR biosensor research [1]. A. C. Huet et al. designed an SPR sensor to detect the flu equine in the food [2]; R. S. Rebe et al. used the SPR technique to image the antibiotics in the milk [3]; Y. Song et al. designed a PH-BSA (bovine serum albumin) labeled SPR immune sensor to measure the phosmet concentration in the agricultural products [4]; Y. Shevchenko et al. manufactured the first SPR biosensor based on the tilted fiber Bragg grating (TFBG) and demonstrated the DNA and protein sensing capability of this biosensor [5]; in another work of Y. Shevchenko et al. [6], the TFBG-SPR biosensor was used to monitor the cell activities in real time, which was proven to be reusable.

To conduct the study on single-nucleotide polymorphism (SNP), we fabricated a TFBG-SPR biosensor by coating a thin layer of gold on the surface of the TFBG-SPR. The sensing principles of the TFBG-SPR biosensor and the experimental process are illustrated in detail in this paper. The experiment was mainly consistent with the treatment

Received: 18 November 2014 / Revised version: 21 December 2014

(C) The Author(s) 2015. This article is published with open access at Springerlink.com DOI: 10.1007/s13320-015-0232-5

Article type: Regular 
of the active biomolecule, self-assemble of the sensing film, and DNA hybridization test. Actually, the activity and structure of the molecules can be protected by using a TFBG-SPR biosensor which is relatively stable and has a good repeatability.

\section{Sensing principles}

Surface plasmon resonance sensors respond to any change in the dielectric environment. The TFBG-SPR can not be used as a sensor of detecting the biological molecule. It will be improved to be a SPR biosensor which can realize the specificity of the specific molecular recognition by coating the sensitive biological membrane outside the active metal deposit layer. Dynamically monitoring the combination of bio-sensitive membrane and specific biological molecules as well as inferring whether the analyst contains some kind of specific DNA molecule can be implemented through observing the transmission spectrum resonance of the TFBG-SPR biomolecular probe sensor. Figure 1 shows the structure of the TFBG-SPR biosensor molecule, and the detection principle of the biological molecular sensor is presented in Fig. 2.

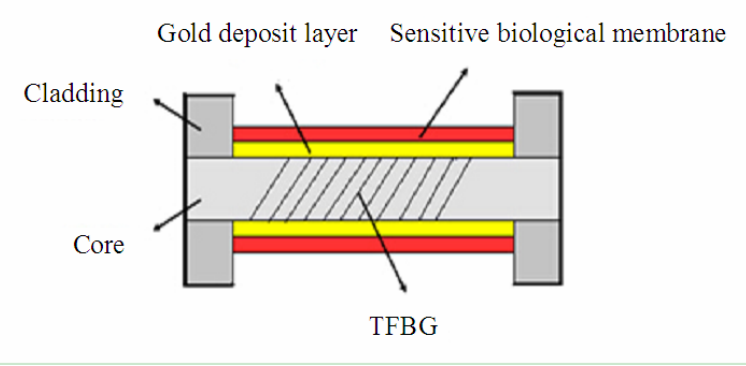

Fig. 1 Structure of the TFBG-SPR biological molecule.

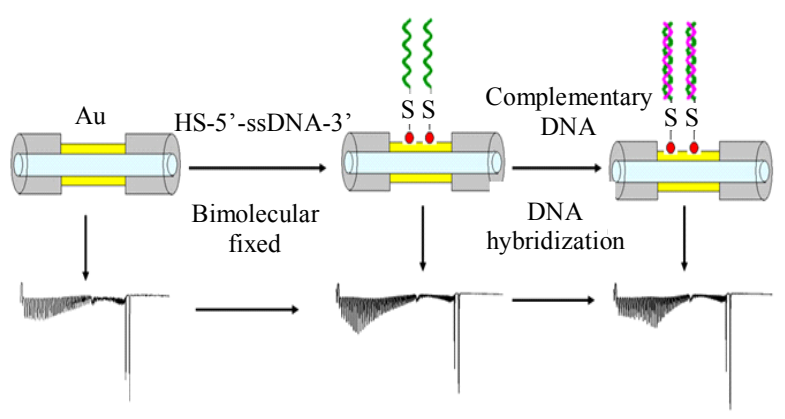

Fig. 2 Detection principle of the biological molecular sensor.

\section{Experimental process}

The main components of the TFBG-SPR biological molecular probe experiment are the processing of bio-sensitive materials and self-assembly fixed sensitive membrane, detecting effect of the adhesion of sensitive materials, and testing DNA hybridization. The reagents and equipments required in the experiment are given in Tables 1 and 2.

Table 1 Reagents required in the experiment.

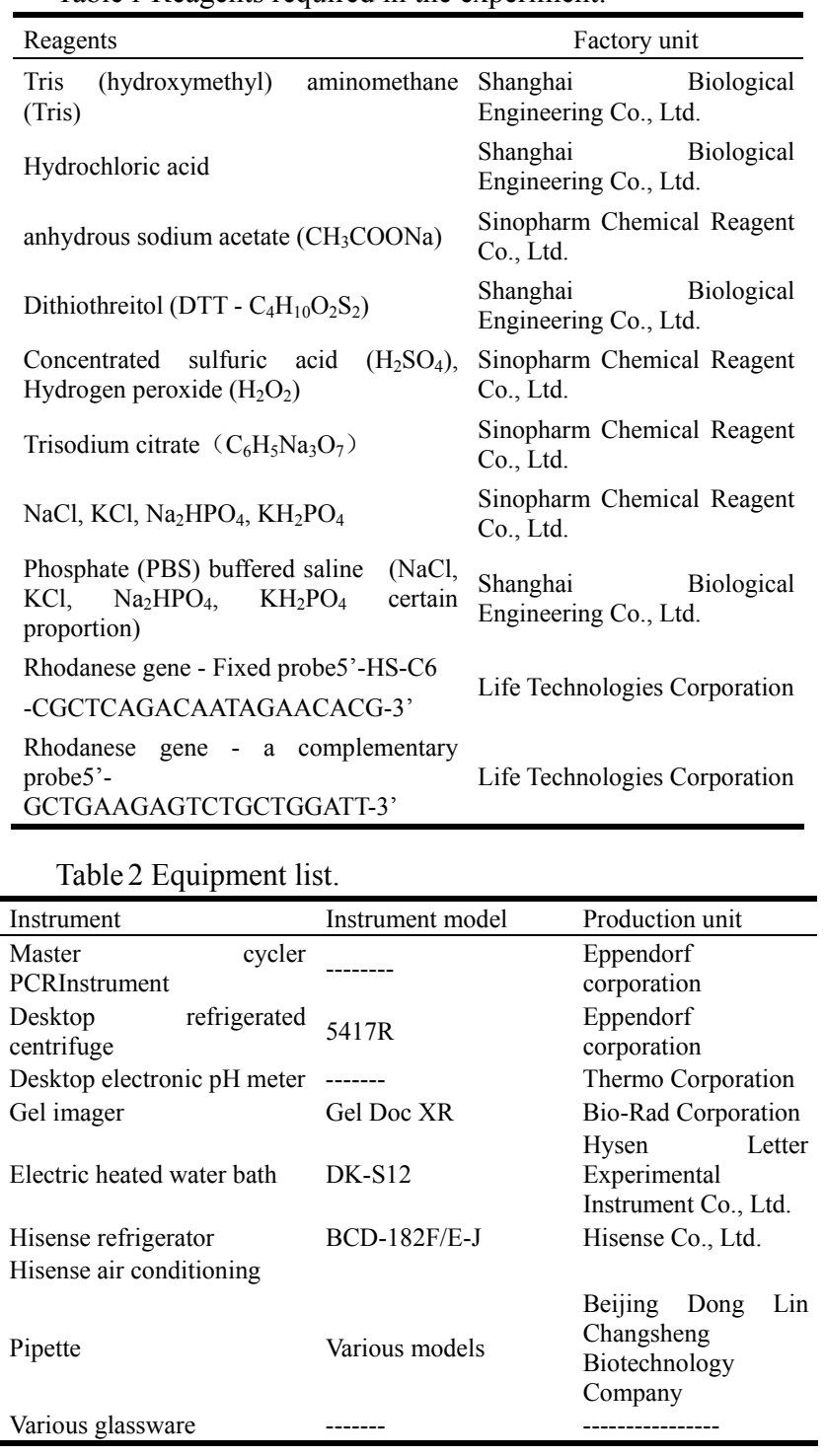

\subsection{Processing of biological sensitive materials}

\subsubsection{Selection of biological sensitive materials}

The material of the specific biological sensitive 
layer was selected for thiocyanate enzyme gene of acidophilic sulfur thermophilic bacillus. Adding modified thiol to the thiocyanate enzyme gene can make it to be a sulfhydryl probe, which can realize the specific identification of complementary DNA. The principle of the combination of the nanogold layer and the DNA probe is presented in Fig. 3.

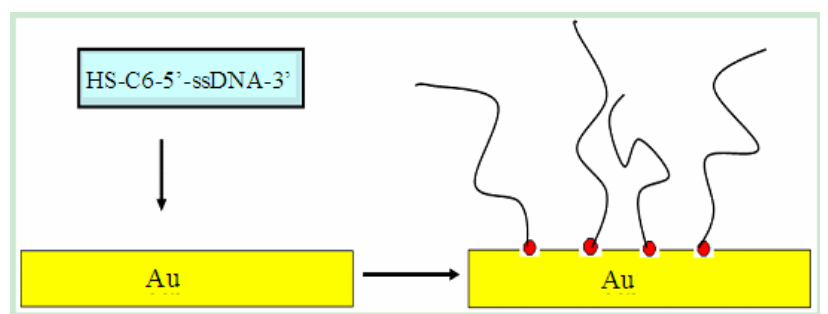

Fig. 3 DNA probe combines with the nanogold film.

\subsubsection{Early period of the biological sensitive materials}

The oxidation of thiol could be prevented by making the thiol end into disulfide bond when the sulfhydryl probe was fabricated. Before being used in the experiment, the disulfide bond was reduced into thiol by the application of DL-Dithiothreitol, etc. Figure 4 shows the molecular structure of the polymorphic DNA.

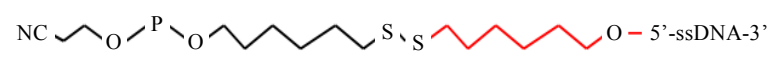

Fig. 4 Molecular structure of polymorphic DNA.

\subsection{Self-assembly fixed sensitive membrane}

The purpose of fixing the bio-sensitive membrane is to keep the enzyme or free cells in a certain area, maintaining its own activity and reusability. The specificity of the biosensor is mainly decided by the bio-sensitive materials, and the adhesion of sensitive materials is an important aspect of the biosensor performance indicators. The thiol self-assembly membrane method was chosen to make the fixed bio-sensitive membrane to achieve the best effect in the experiment. Self-assembled membrane (SAMS) is a kind of ordered membrane, which is relatively stable and formed by the interaction of the intermolecular and molecular substrates. It has many good features such as regular arrangement, thermodynamic stability, and high bulk density [12].

Exposure to the piranha solution $\left(\mathrm{H}_{2} \mathrm{SO}_{4}\right.$ : $\mathrm{H}_{2} \mathrm{O}_{2}=3: 1$ proportion), distilled water and ethanol cleaned the surface of the biomolecular probe sensor from cellular residues before the experiment.

The two gold-plated TFBGs (numbered A and B) were fixed on a glass dish and then placed in a thermostatic water bath with a temperature of $24^{\circ} \mathrm{C}$. Figure 5 presents the experimental apparatus, and the temperature of the indoor air should be $24{ }^{\circ} \mathrm{C}$ in order to reduce the impact on the experiment.

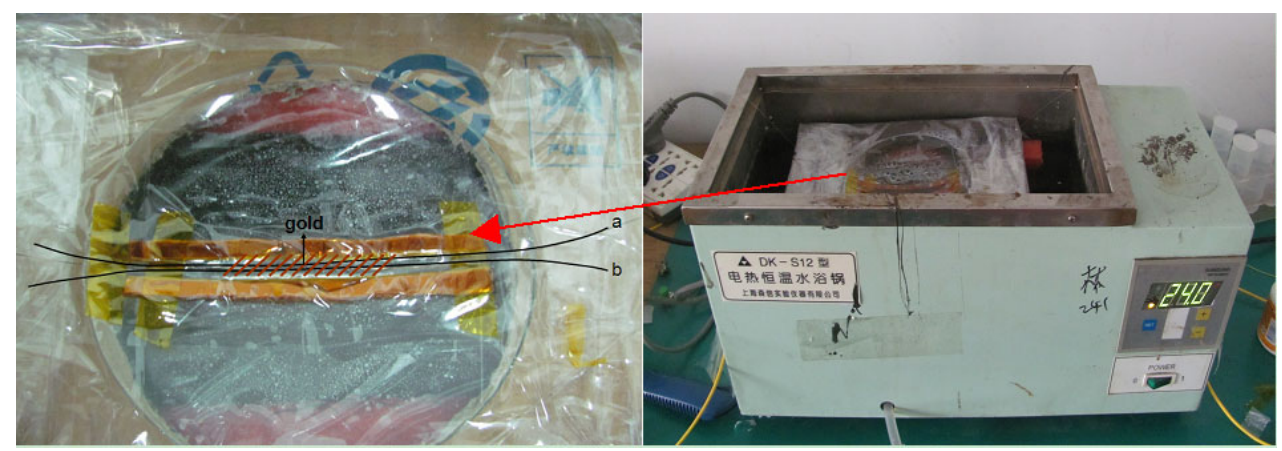

Fig. 5 Experimental device.

The configured solution of the DNA gene probes was injected into the strip groove with a pipette and then exposed the two gold-plated TFBGs to react with the complementary DNA in the solution above $5 \mathrm{~h}$. The changes what happened to the transmission spectrum of the TFBG numbered $B$ should be observed in the molecular modification process. After the entire reaction was completed, the TFBG numbered A was used to verify the feasibility of fixing probe methods. 


\subsection{Detection of self-assembled fixed effect}

\subsubsection{Biological methods to verify the probe fixed effects}

The phosphate buffer saline (PBS) was used to rinse the TFBG probe numbered $A$ to remove shrouded DNA molecules. The modified fiber should be immersed into the $520 \mu \mathrm{L} 0.1 \mathrm{M}$ Tris- $\mathrm{HCl}$ containing $8 \mathrm{mg}$ DL-Dithiothreitol standing for half an hour at the room temperature. A portion of the solution with a probe complementary was taken to a primer pair for polymerase chain reaction (PCR) amplification, which was then subjected to electrophoresis to detect whether it contained paired DNA.

If the combination can be accomplished, the solution dealed with the DL-Dithiothreitol will contain some thiol probe primers, which can hybridize with the complementary probe to amplify DNA bands.

The results of the amplification are shown in Fig. 6 and the solid larger circle presents the DNA marker, from right to left for the $8000 \mathrm{bp}, 5000 \mathrm{bp}$, 3000 bp, 2000 bp, 1000 bp, 750 bp, 500 bp, 250 bp, $100 \mathrm{bp}$ (bp represents base pair, namely base pairs), characterizing the length of the DNA. The dotted minor circle is amplified by the imaginary bands, indicating that the modification of the DNA probe method is feasible.

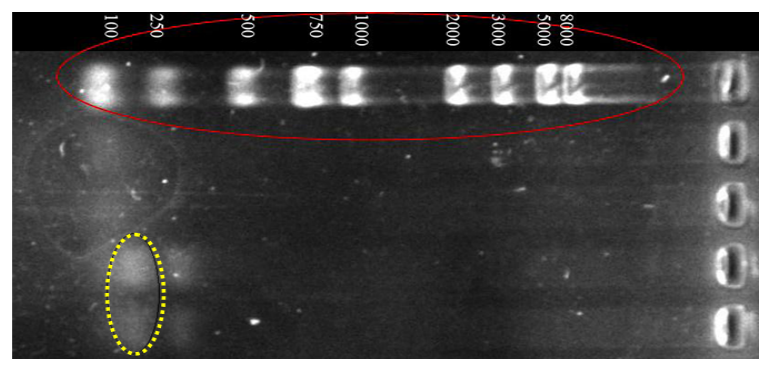

Fig. 6 Results of DNA amplification.

\subsubsection{Optical methods to verify the feasibility of fixing probe method}

The experimental setup for detecting the changes in the transmission spectrum and in the self-assembly process of the TFBG numbered B is presented in Fig. 7. The spectral power problem was ignored to facilitate the observation of changes effectively. The results of the transmission spectrum at $0 \mathrm{~min}, 10 \mathrm{~min}, 15 \mathrm{~min}, 20 \mathrm{~min}, 26 \mathrm{~min}, 45 \mathrm{~min}$, and $57 \mathrm{~min}$ are listed down one by one in Fig. 7 (a), indicating the effective refractive index of the outer cladding changed, and the adhesion of the self-assembled monolayer has been conducting as time went on.

The changes in the TFBG resonant wavelength in the whole self-assembly process are shown in Fig. 7(b). The resonance peak of the transmission spectrum almost did not shift in the beginning $10 \mathrm{~min}$, and its shift accelerated after $10 \mathrm{~min}$, which indicated the speed-up of the reaction. However, after $57 \mathrm{~min}$, it almost did not shift any more.

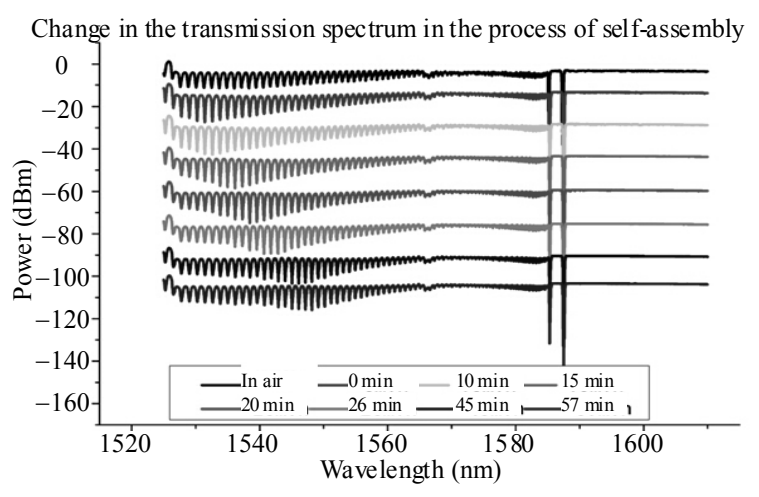

(a)

Change in the transmission spectrum in the process of self-assembly

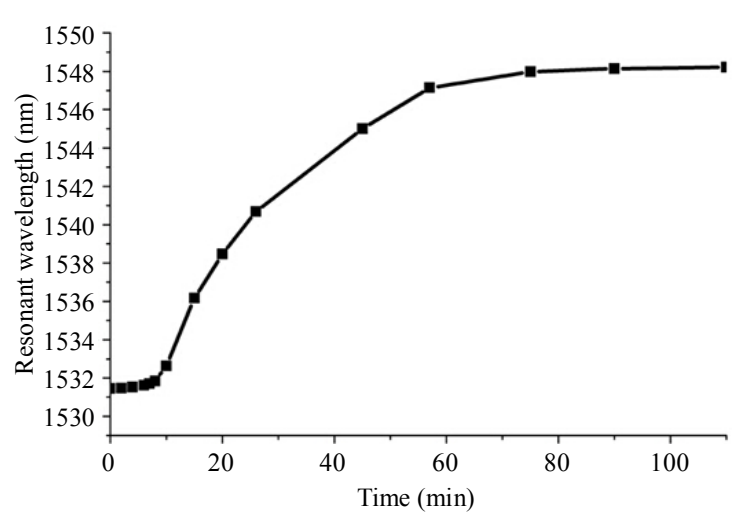

(b)

Fig. 7 Changes in the transmission spectrum and the central wavelength in the process of self-assembly: (a) changes in the transmission spectrum and (b) changes in the central wavelength. 


\subsection{DNA hybridization experiments}

The important part of the surface plasmon biosensor detection is molecular specificity recognition of the TFBG with a layer of gold. The effective refractive index of the gold membrane will change when the self-assembly of gene probes is combined with complementary gene probes, causing shifts in the transmission spectrum of the TFBG resonance.

Before the experiment, the complementary DNA (thermophilic acidophilic bacillus thiocyanate gene) solution with the concentration of $0.1 \mathrm{mmol} / \mathrm{L}$ and aqueous solution $(6 \times \mathrm{SSC}, 20 \mathrm{mmol} / \mathrm{L}$ PBS $)$ should be prepared. After fixing the TFBG with the gene probe on the glass dish, the glass dish was placed into a constant temperature water bath at the temperature of $30{ }^{\circ} \mathrm{C}$ for $10 \mathrm{~min}$. Then an aqueous solution of complementary genes should be injected into recesses to observe the changes in the TFBG transmission spectrum. The experimental equipments of the hybridization are presented in Fig. 8.

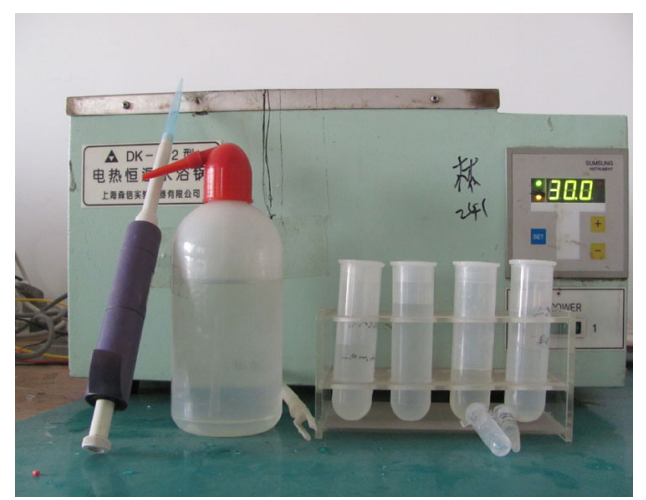

Fig. 8 Experimental equipment used in the nucleic acid hybridization.

Figures 9(a) and 9(b) show the changing process of the TFBG transmission spectra as the complementary solution with a concentration of $0.1 \mathrm{mmol} / \mathrm{L}$ and also illustrate that the formant of the TFBG transmission spectra gradually shifted to the larger wavelength direction as time went on.

The resonant wavelength changed with time in the nucleic acid hybridization process. Figure 10 shows that in the period of $25 \mathrm{~min}-46 \mathrm{~min}$ the change in the resonant wavelength was significant, and then the resonance wavelength shifted slower from $45 \mathrm{~min}$ to $100 \mathrm{~min}$. After the reaction was conducted for $100 \mathrm{~min}$, the hybridization reaction has been completed substantially with no change in the resonant wavelength.

Change in the transmission spectrum in the process of acid hybridization

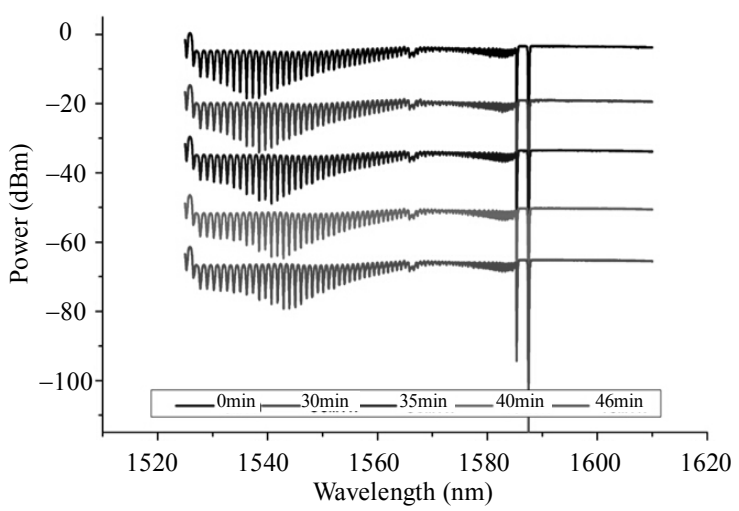

(a)

Change in the transmission spectrum in the process of acid hybridization

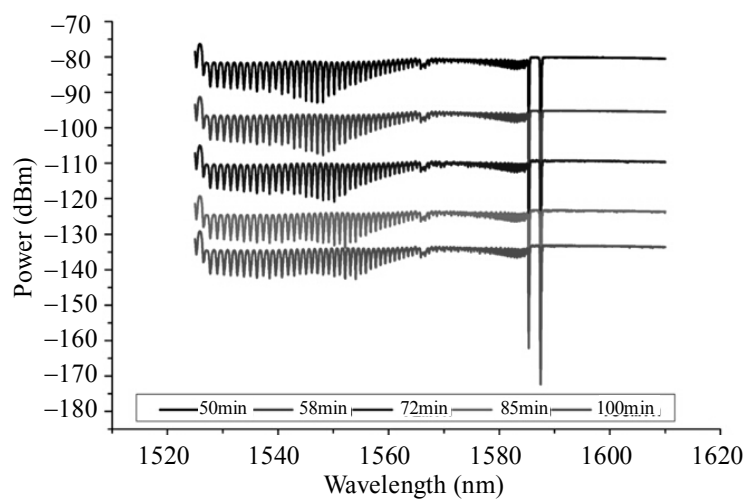

(b)

Fig. 9 Change in the transmission spectrum in the process of nucleic acid hybridization: (a) $0 \mathrm{~min}-46 \mathrm{~min}$ and (b) $50 \mathrm{~min}-$ $100 \mathrm{~min}$.

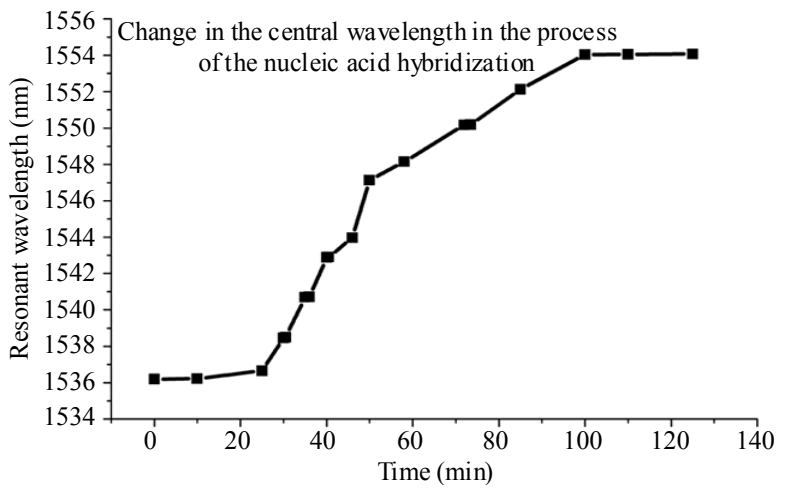

Fig. 10 Change in the central wavelength in the process of the nucleic acid hybridization (the solution concentration of the complementary probe was $0.1 \mathrm{mmol} / \mathrm{L}$ ). 
After using the same method to regulate the probe on the TFBG with the same parameters, the hybridization experiments could be conducted. Before the experiment, the complementary DNA solution with a concentration of $0.05 \mathrm{mmol} / \mathrm{L}$ should be prepared, and the experimental procedure was the same when the concentration was $0.1 \mathrm{mmol} / \mathrm{L}$. The changes in the TFBG transmission spectrum in the hybridization process are presented in Figs. 11(a) and 11(b). Figure 12 shows the resonance of the TFBG corresponding to the wavelength variation.

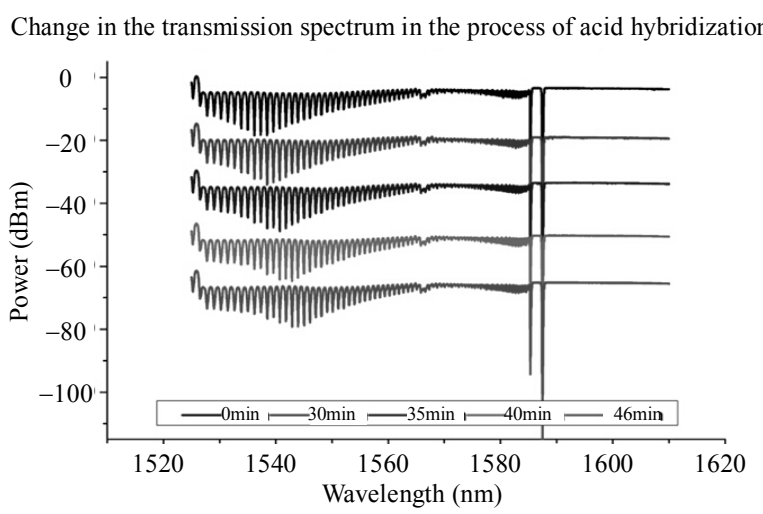

(a)

Change in the transmission spectrum in the process of acid hybridization

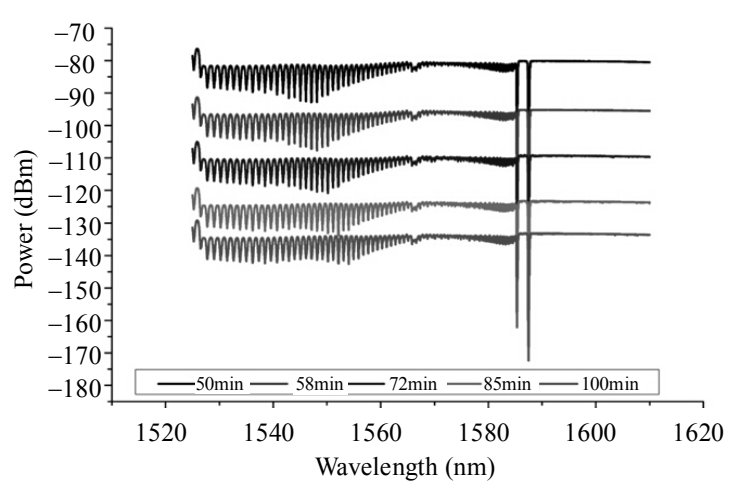

(b)

Fig. 11 Change in the transmission spectrum in the process of nucleic acid hybridization: (a) $0 \mathrm{~min}-46 \mathrm{~min}$ and (b) $60 \mathrm{~min}$ $-100 \mathrm{~min}$.

The results of the hybridization experiment when the concentration of the complementary DNA probe was $0.1 \mathrm{mmol} / \mathrm{L}$ were basically the same as the results with a concentration of $0.05 \mathrm{mmol} / \mathrm{L}$. The resonance of the TFBG transmission spectra when the concentration was $0.1 \mathrm{mmol} / \mathrm{L}$ DNA changed faster than those with a concentration of $0.05 \mathrm{mmol} / \mathrm{L}$.

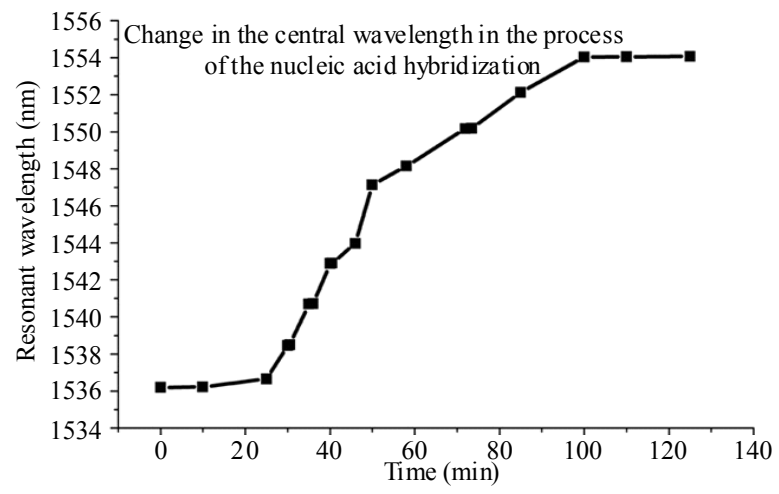

Fig. 12 Change in the central wavelength in the process of nucleic acid hybridization (the solution concentration of the complementary probe was $0.05 \mathrm{mmol} / \mathrm{L}$ ).

As the DNA hybridization experiments have shown, the TFBG resonant wavelength gradually increased. The results indicated that the hybridization reaction changed the effective refractive index of the gold membrane, meanwhile illustrated the feasibility of using the biomolecular probe sensor based on the TFBG surface plasma resonance to detect the specific DNA.

\section{Conclusions}

The study and experiments on the biomolecular probe sensor based on the TFBG surface plasma resonance were presented. Firstly, the self-assembly method and the principles of hybridization were introduced, and then the process of fixing self-assembly of molecular probes and changes in the transmission spectrum and resonant wavelength of the TFBG throughout the process were described. Secondly, the hybridization of the fixed probe with the complementary probe was conducted, and the dynamic process of the resonant wavelength was observed. The results indicated that the hybridization changed the effective refractive index of the golden layer, meanwhile illustrated the feasibility of the biomolecular probe sensor based on the TFBG surface plasma resonance for detecting the specific molecule. 


\section{Acknowledgment}

This work was financially supported by China NSFC (No. 61271073 and 61473175).

Open Access This article is distributed under the terms of the Creative Commons Attribution License which permits any use, distribution, and reproduction in any medium, provided the original author(s) and source are credited.

\section{References}

[1] Q. Jiang, D. D. Lv, M. H. Yu, L. M. Kang, and J. Ouyang, "The experiment research on solution refractive index sensor based on tilted fiber Bragg grating," Spectroscopy and Spectral Analysis, 2013, 33(12): 3425-3431.

[2] A. C. Huet, C. Charlier, G. Singh, S. B. Godefroy, J. Leivo, M. Vehniäinen, et al., "Development of an optical surface plasmon resonance biosensor assay for (fluoro) quinolones in egg, fish, and poultry meat," Analytica Chimica Acta, 2008, 623(2): 195-203.

[3] R. S. Rebe, M. G. Bremer, W. Haasnoot W, and W. Norde, "Label-free and multiplex detection of antibiotic residues in milk using imaging surface plasmon resonance-based immunosensor," Analytical chemistry, 2009, 81(18): 7743-7749.

[4] Y. Song, M. Liu, and S. Wang, "Surface plasmon resonance sensor for phosmet of agricultural products at the ppt detection level," Journal of Agricultural and Food Chemistry, 2013, 61(11): 2625-2630.

[5] Y. Shevchenko, D. Blair, M. C. Derosa, and J. Albert, "DNA target detection using gold-coated tilted fiber Bragg gratings in aqueous media," in Conference on Lasers and Electro-Optics, 2008 and 2008 Conference on Quantum Electronics and Laser Science, San Jose, pp. 1-2, 2008.

[6] Y. Shevchenko, G. Camci-Unal, D. F. Cuttica, M. R. Dokmeci, J. Albert, and A. Khademhosseini, "Surface plasmon resonance fiber sensor for real-time and label-free monitoring of cellular behavior," Biosensors and Bioelectronics, 2014, 56: 359-367. 